\title{
Strength of the interatomic potential derived from Angular Scans in LEIS
}

\author{
D. Primetzhofer ${ }^{a^{*}}$, S.N. Markin ${ }^{a}$, M. Draxler ${ }^{a}$, R. Beikler ${ }^{b}$, E. Taglauer ${ }^{b}$ and P. Bauer ${ }^{2}$
}

${ }^{a}$ Institut für Experimentalphysik, Johannes Kepler Universität Linz, A-4040 Linz, Austria.

${ }^{b}$ Max-Planck-Institut für Plasmaphysik, EURATOM Association, D-85748 Garching bei München, Germany.

\begin{abstract}
Angular scans were performed for a $\mathrm{Cu}(100)$ single crystal and $\mathrm{He}^{+}$ions. The results were compared to MARLOWE, KALYPSO and FAN simulations to obtain information on the interaction potential. The influence of the used evaluation procedure on the deduced scattering potential was investigated. The scattering potential is found to be weaker than what is predicted by an uncorrected TFM potential. It was found that the use of a single screening correction factor is applicable in a wide range of impact parameters. It is further shown that selection of single scattering trajectories and a limitation of information depth to the surface layers is possible for neutral and charge integrated spectra.
\end{abstract}

\section{PACS numbers:}

34.20.Cf, 34.50.s, 68.49.Sf, 79.20.Rf, 61.85.+p

\section{Keywords:}

Low energy ion scattering, single crystal, $\mathrm{Cu}(100)$, screening length, interaction potential, Monte-Carlo simulations, Molecular Dynamics simulations

\footnotetext{
*daniel.primetzhofer@jku.at
}

\section{Introduction}

Low-energy ion scattering (LEIS) is a commonly used tool for analysis of composition and structure of solid surfaces [1], [2]. Usually, noble gas ions are used as primary particles. If only scattered ions are detected, the information depth is limited to a few atomic layers, due to effective neutralization [3]. Ions are usually detected by use of an electrostatic analyzer (ESA-LEIS). By applying the time-of-flight (TOF) technique and post acceleration of ions that survived the scattering process, backscattered ions and neutrals can be analyzed separately.

In the LEIS regime (energy range 1 $10 \mathrm{keV}$ ) the scattering potential, e.g. ThomasFermi-Molière potential (TFM) [4], is given by a screened Coulomb potential

$$
\mathrm{V}(\mathrm{r})=\frac{\mathrm{Z}_{1} \mathrm{Z}_{2} \mathrm{e}^{2}}{\mathrm{r}} \cdot \Phi\left(\frac{\mathrm{r}}{\mathrm{a}}\right),
$$

with $Z_{1}$ and $Z_{2}$ the atomic numbers of ion and scattering centre, respectively, and $\Phi(r / a)$ the screening function with the characteristic screening length a [5], [6]. Usually, the screening length is expressed as $a=c_{a} \cdot a_{F}$, with $a_{F}$ the Firsov screening length [7], and $c_{a}$ a correction factor, which is typically determined empirically for a given projectiletarget combination. This correction just introduces a scaling factor for the potential: $\mathrm{c}_{\mathrm{a}}$ $<1$ corresponds to a smaller value of $\Phi$ and thus to a weaker potential in the complete range of impact parameters. Consequently, for a given impact parameter $\mathrm{c}_{\mathrm{a}}<1$ leads to a smaller scattering angle and for a given scattering angle, to a smaller scattering cross section [8]. It is obvious that for quantitative 
surface composition and structure analysis the exact strength of the scattering potential has to be well known since it directly influences resulting backscattered intensities and their angular distribution. Another important fact is that the results presented in [9] for a wide range of interaction distances imply that this scaling may not be suitable to reproduce the entire scattering potential.

For single crystalline targets the spectra obtained in a TOF-LEIS experiment show a characteristic dependence on the orientation and on the crystal structure of the sample. This is due to angular dependent shadowing of subsurface layers and blocking of scattered contributions from deeper layers [10]. For a sample of well known structure, information on the interaction potential can be extracted, if one records the yield of backscattered particles as a function of the scattering geometry and compares the results to computer simulations obtained from different codes. In FAN [ 11], [ 12 ] the backscattered intensity is calculated in a fast way by starting the calculation of possible inand outgoing trajectories at the position of the scattering centre. MARLOWE [13] is a Monte Carlo code used for trajectory simulations. KALYPSO [14] is a molecular dynamics program. By change of the input parameters in the simulation, e.g. $c_{a}$ or inelastic energy loss, the calculated angular scans can be fitted to the experiment. The best fit of position and shape of the observed maxima and minima finally yields the interaction potential, expressed in terms of $c_{a}$. Values in the range from 0.5 to 1 have been reported [8], [10], [15], [16], [17], [18], [19], [20] even for the identical ion-target combination. Of course, the described procedure can be carried out in the reverse way, deducing an unknown surface structure by comparison of the experimental yield to simulations for different structural models.

In any case the situation is actually more complex due to the fact that there are different possibilities to define the relevant information of the experimental yield, to which finally the simulations are compared. This is of crucial importance since in LEIS not only the total observed intensity but also the shape of the spectrum exhibits a strong dependence on the angle of incidence, $\alpha$, and the azimuth orientation of the scattering plane, $\varphi$. In particular, in TOF-LEIS spectra for detected neutrals, a background that originates from multiple scattering and contributions from deeper layers is observed. In [21] the origin of different features observed in TOF-LEIS spectra, i.e. the intensities of single scattering surface peak and background is analyzed. In addition, it was shown how highly sensitive the resulting intensities depend on evaluation procedures in angular scans. Furthermore, it was demonstrated that for a simple system, i.e. $\mathrm{He}^{+}$and $\mathrm{Cu}(100)$, it is possible to enhance significantly the surface sensitivity of angular scans of scattered neutrals. This was achieved by background subtraction, such that only single scattered particles are selected.

In the present work a correction factor for the TFM scattering potential is obtained from comparison of experimental angular scans to simulations using different software packages. Data are deduced in different ways from the same experimental dataset in order to investigate if possible systematic errors may be introduced by different evaluation procedures. By a comparison of the experimental results obtained in [21] to the different simulations it is shown how to evaluate a spectrum properly in order to extract the signal originating from the topmost atomic layers only. This affects significantly surface structure and composition analysis using TOF-LEIS. Furthermore, it is checked whether by one screening correction factor it is possible to describe the scattering potential for the entire range of impact parameters of interest. Advantages and disadvantages of the simulation codes used are discussed.

To disentangle scattering potential and structure, the interaction of $\mathrm{He}^{+}$and $\mathrm{Cu}(100)$ is studied, since this surface is unreconstructed and the small surface relaxations are well known from investigations using different techniques [22].

\section{Experimental setup}

The experiments were performed using the Time-Of-Flight- (TOF-) LEIS setup ACOLISSA [23] with a scattering angle $\theta$ of $129^{\circ}$ and a detector acceptance angle of $0.92^{\circ}$. The angular precision of the manipulator is $\pm 0.1^{\circ}$ and $\pm 0.2^{\circ}$ for polar and azimuth scans, respectively. The system is typically operated at a time resolution set to 10 to 25 ns corresponding to an energy resolution of 1 to $5 \%$ for $\mathrm{He}^{+}$ions at $3 \mathrm{keV}$. A post acceleration voltage can be applied along part of the flight path between sample and detector to separate backscattered ions from neutrals. The primary beam current is set between 25 to $100 \mathrm{nA}$ in full beam mode, yielding 5 to $20 \mathrm{pA}$ in the chopped beam mode, which makes TOF-LEIS virtually non-destructive. The beam current remains constant to within $10 \%$ after thermal equilibration ( 2 h). At normal incidence, the beam spot on the sample was found to be smaller than $1 \mathrm{~mm}$ in diameter. From this the "safe" range of incident angles follows (angle of incidence $\alpha<65^{\circ}$, with respect to the surface normal) ensuring that 
the whole irradiated spot is visible for the detector.

The measurements were performed on a $\mathrm{Cu}(100)$ single crystal surface. ${ }^{4} \mathrm{He}^{+}$ions with a primary energy of $3 \mathrm{keV}$ were used as projectiles. Azimuth scans $Y(\varphi)$ were measured in an angular range of $140^{\circ}$.

The sample was prepared by repetitive sputtering - annealing cycles, performed with $3 \mathrm{keV} \mathrm{Ar}^{+}$ions and subsequent heating to $\sim 650 \mathrm{~K}$. Surface purity and crystal structure of the sample were checked by Auger electron spectroscopy (AES) and lowenergy electron diffraction (LEED) (Fig.2), respectively.

Results and discussion:

In LEIS experiments, for an unreconstructed (100) surface of an fcc crystal like $\mathrm{Cu}(100)$ only the two outermost atomic layers contribute to single scattering at normal incidence $\left(\alpha=0^{\circ}\right)$ due to shadowing of deeper layer atoms. The scattering angle $\Theta=129^{\circ}$ permits to make full use of the crystal symmetry in these azimuth scans. This is because of two factors: first, the primary beam is aligned with a major crystal axis ([100]), featuring excellent channeling conditions, and second, the exit angle is close to $45^{\circ}$, leading to angular dependent blocking of the second layer scattering contributions at sufficiently low energies. In particular, at $0^{\circ}$ and $45^{\circ}$ azimuth the detection beam line is almost parallel to the [110] and [111] crystallographic directions, respectively. This further suppresses contributions from multiple scattering in deeper layers. Exploiting these advantages, all azimuth scans presented here were recorded for normal incidence and $3 \mathrm{keV} \mathrm{He}^{+}$ions, which also allows one for a direct comparison with [15]. Fig.1a presents a top view of the (100) fcc surface. Fig.1b shows the relevant scattering planes for which single scattering from the first and second layer only with minimum perturbation from multiple scattered and deeper layer contributions can be expected. Two typical energy converted charge integrated TOF-spectra recorded in double alignment and a random geometry for $3 \mathrm{keV} \mathrm{He}{ }^{+}$and $\mathrm{Cu}(100)$ are shown in Figs.2a and $2 \mathrm{~b}$ together with a LEED pattern which shows a clear $(1 \times 1)$ cubic pattern with sharp spots indicating a good crystal surface quality. The surface peak observed for both spectra is expected to originate from single scattering in the outermost two atomic layers only. Note, that the background due to multiple scattering and to contributions from deeper layers is enormously enhanced in the spectrum at random

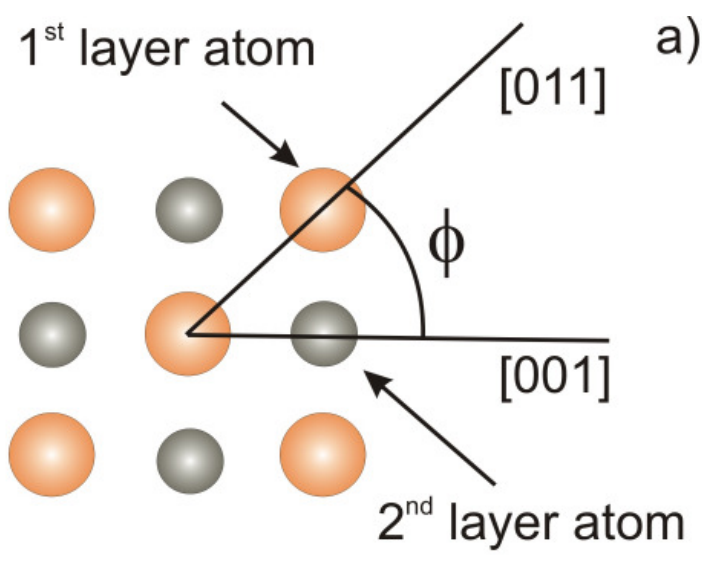

b)
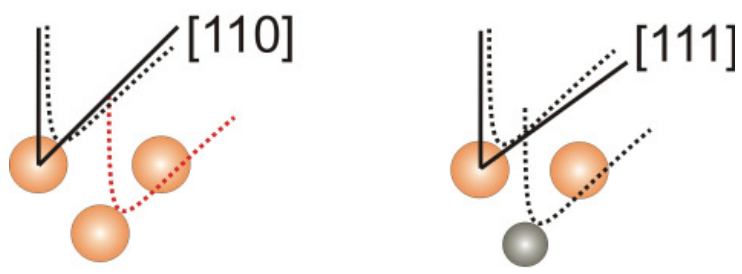

in-plane atoms:

out-of-plane atoms: possible trajectories: impossible trajectories:

Fig.1: a) Top-view of a (100) fcc-surface. b) Scattering planes formed by the surface normal and the crystallographic directions indicated in a). Also shown are possible trajectories for single scattering processes.

In literature, hardly any information is found on such details as the definition of "peak intensity". One might argue that obviously it is most reasonable to integrate the surface peak after background subtraction [21]. However, for a detailed angular scan this may be a time consuming and tedious task. Therefore, we analyze in the following which are the consequences when different evaluation algorithms are applied. One possibility is to integrate a TOF- or energy range corresponding to a predefined region around the surface peak without any background subtraction. Within reasonable limits, three different sizes of the integration window in the TOF spectra were chosen, with a corresponding energy interval between 148 and $247 \mathrm{eV}$ (dark and light grey shaded areas in Fig.2 a) and b, respectively)). The resulting angular scans were normalized and the ratio of the yield of either the small $\left(Y_{\Delta_{-}}\right)$or the large $\left(\mathrm{Y}_{\Delta_{+}}\right)$energy window to the intermediate one $\left(\mathrm{Y}_{\bar{\Delta}}\right)$ (grey shaded) was evaluated; a relative change below $7 \%$ was found, as presented in Fig.3a. Thus, this procedure is rather stable 
and possible systematic errors are small regarding the relative intensities.
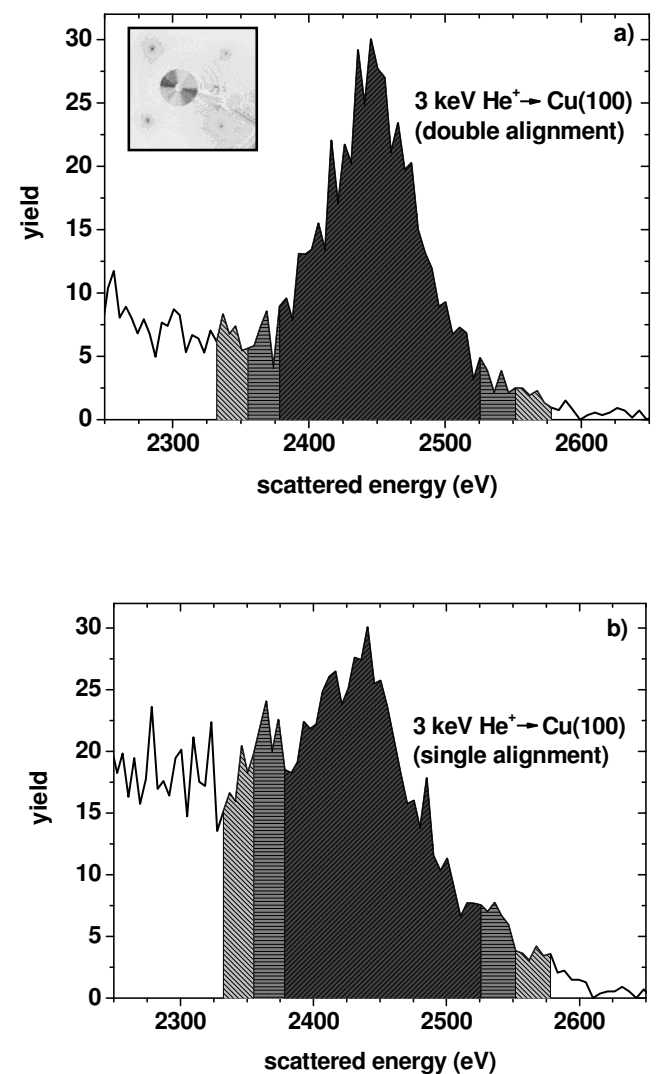

Fig.2: Energy converted TOF-LEIS spectra recorded for $3 \mathrm{keV} \mathrm{He}{ }^{+}$scattered for normal incidence from $\mathrm{Cu}(100)$ : a) in a double alignment direction b) in a random exit direction. The energy windows corresponding to the integrated TOF range are indicated by different shades of grey (see text). The inset in a) shows a LEED pattern obtained for the surface after the preparation routine.

Note, however, that the width of the minimum will change slightly and becomes wider for smaller integration windows as can be seen from the intersection of the curves with the unity line in Fig.3.

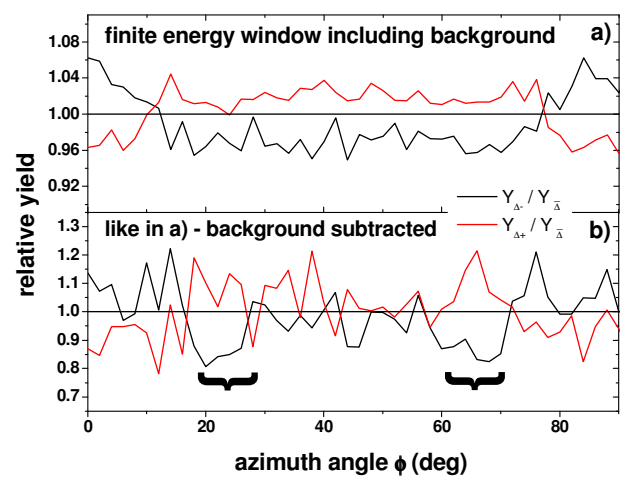

Fig.3: Relative yield obtained from integrating the different energy windows presented in Fig.1: a) for a finite energy window b) for the same energy windows but with subtracted background contributions. The brackets mark the transition area of maximum and minima

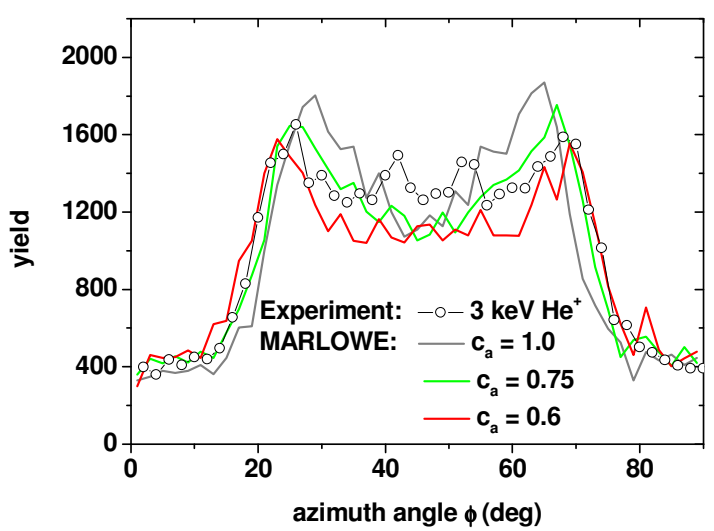

Fig.4: Azimuth scan obtained for $3 \mathrm{keV} \mathrm{He}^{+}$ scattered from $\mathrm{Cu}(100)$ and normal incidence (line + symbols). The experimental data was obtained by integration of an intermediate TOF-window with the width of the surface peak (see Fig1). MARLOWE simulations obtained for different interaction potential strength (lines) and a 18 layer crystal.

Fig. 4 presents the azimuth dependent yield obtained from integrating the grey shaded TOF range. It also contains three different MARLOWE simulations, calculated for a crystal thickness of 18 monolayers, and for three different $c_{a}$ values, i.e. $1,0.75$ and 0.6 . The minimum energy of the simulated projectiles was set to the low energy limit of the experimental integration window. A velocity proportional inelastic energy loss was included in the calculation in order to suppress unrealistic high contributions from deeper layers. The bulk debye temperature $\Theta_{\mathrm{D}}$ was set to $315 \mathrm{~K}$ and isotropic vibrations were assumed. Simulated spectra were normalized in order to fit the experiments.

The angular scans exhibit a pronounced minimum at $0^{\circ}$ and $90^{\circ}$ azimuth, where excellent double alignment conditions are met [24]. The maximum exhibits an internal structure which can also be explained by angular dependent blocking leading to a local minimum in intensity at $45^{\circ}$ where the detection beam line is oriented close to the [111] crystallographic direction. In the calculation the total backscattered intensity originates to a significant fraction from deeper layers, e.g. for a screening correction factor $\mathrm{C}_{\mathrm{a}}$ $=0.75$ the contribution from layers $4-7$ is $\sim 15 \%$ and from layers $8-12$ is $\sim 3 \%$ of the total yield. The relative contribution in the transition area of minima to maxima is significantly enhanced to $\sim 20 \%$ for layer No's. $4-7$. This is reasonable since in this azimuth orientation no suppression of deeper layer scattered particles due to blocking can be expected. If as a criterion for the best fit overall agreement 
in the whole angular range is taken, an r-factor analysis clearly favors $c_{a}=0.75$ since for this $c_{a}$ value the relative intensities in the plateau region are best reproduced. This is in good agreement with the results obtained in [20]. Note, that if one defines as a criterion that the angular positions of the onsets of the maxima coincide $c_{a}=0.6$ is favored. Furthermore, although the intensity ratio of minima and maximum is wrong, $c_{a}=0.6$ also yields a good reproduction of the shape of the plateau; this might be taken as an indication that an even lower screening correction value is required to reproduce the observed features, i.e. the fine structure of the plateau. The main argument for using the angular width of the maximum for the best fit is that it is directly related to the potential via the width of the shadow cones of surface atoms that block contributions scattered from deeper layers.

This results show a distinct difference to the extraordinary low screening length correction $\mathrm{c}_{\mathrm{a}}=0.53$ obtained in a CAICISS TOF-experiment for $3 \mathrm{keV} \mathrm{He}^{+}$and $\mathrm{Cu}(100)$ and consequent comparison to FAN simulations [15]. In our investigations we have found, by comparison to experimental and MARLOWE results, that the FAN code is typically not suitable to reproduce absolute experimental intensities, i.e. ratios of maxima and minima, especially in the case of a crystal with a z-extension of more than one lattice constant. However, angular positions of maxima and minima where usually reproduced in a correct way, if a slightly reduced screening correction ( 15\%) was implemented with respect to the MARLOWE simulations.

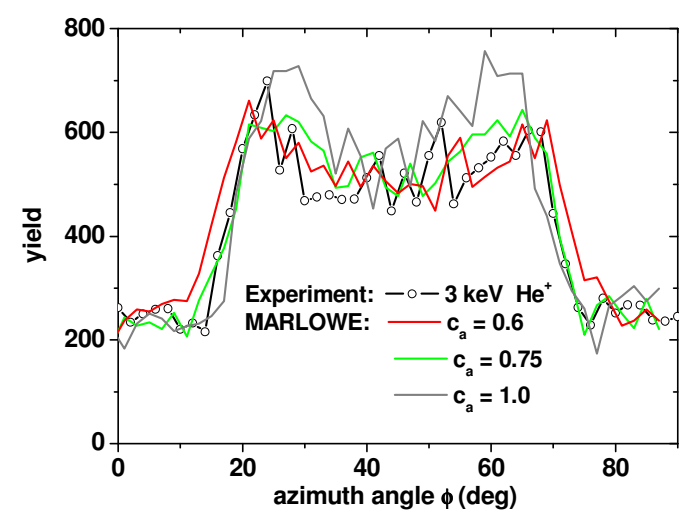

Fig.5: Azimuth scan obtained for $3 \mathrm{keV} \mathrm{He}^{+}$ scattered from $\mathrm{Cu}(100)$ and normal incidence (line + symbols). The experimental data was obtained by integration of an intermediate TOF-window with the width of the surface peak and subtraction of a linear decreasing background (see Fig1). MARLOWE simulations obtained for different interaction potential strength (lines) and a model crystal that consists of only two layers.
By applying linear background subtraction according to [21] to the surface peak, one obtains an experimental angular dependence as presented in Fig. 5. It is expected that this procedure leads to a selection of single scattering trajectories from the two outermost layers only. Due to the lower yield, the statistical spread of the presented experimental data is larger. One might expect that subtraction of the background would result in a pronounced dependence of the scattered intensity on the window size. Explicitly, a change in the size of the integration window by $66 \%$ resulted in a maximum intensity change $<20 \%$ (see Fig.2b). Also, the width of the maxima (FWHM) in the angular scan is not changed significantly. In contrast, the intensity and therefore the shape of the maxima close to the edges (indicated in Fig.3b by brackets) is found to change markedly when the width of the integration window is altered (as indicated in Fig.2a and $2 b$ ). For the widest and the narrowest integration window, the shapes of the maxima could not be reproduced in any simulation which shows that also a reasonable choice for the width of the integration window is essential for a good match with simulations.

In this special case described, simulations can be performed for a thin target consisting of only two layers of a (100) oriented crystal surface. This permits an easy comparison of the results obtained from different simulation codes for different screening length correction factors within reasonable computation time.

Fig. 5 also contains the result of MARLOWE simulations obtained for the same $c_{a}$ values as in Fig. 4, but now for the outermost two atomic layers only. In Figs. 6 and 7 the same experimental dataset is compared to simulations of scattering from the first two monolayers using the molecular dynamics simulation KALYPSO and the FAN code. Simulations with KALYPSO were performed with and without including inelastic energy loss. Only very minor change of the resulting angular scans was observed. $\Theta_{D}$ was set to $300 \mathrm{~K}$. Neglecting thermal vibrations results in unrealistic sharp features in the spectra - however a change of $10 \%$ in $\Theta_{D}$ has only a very small effect. 

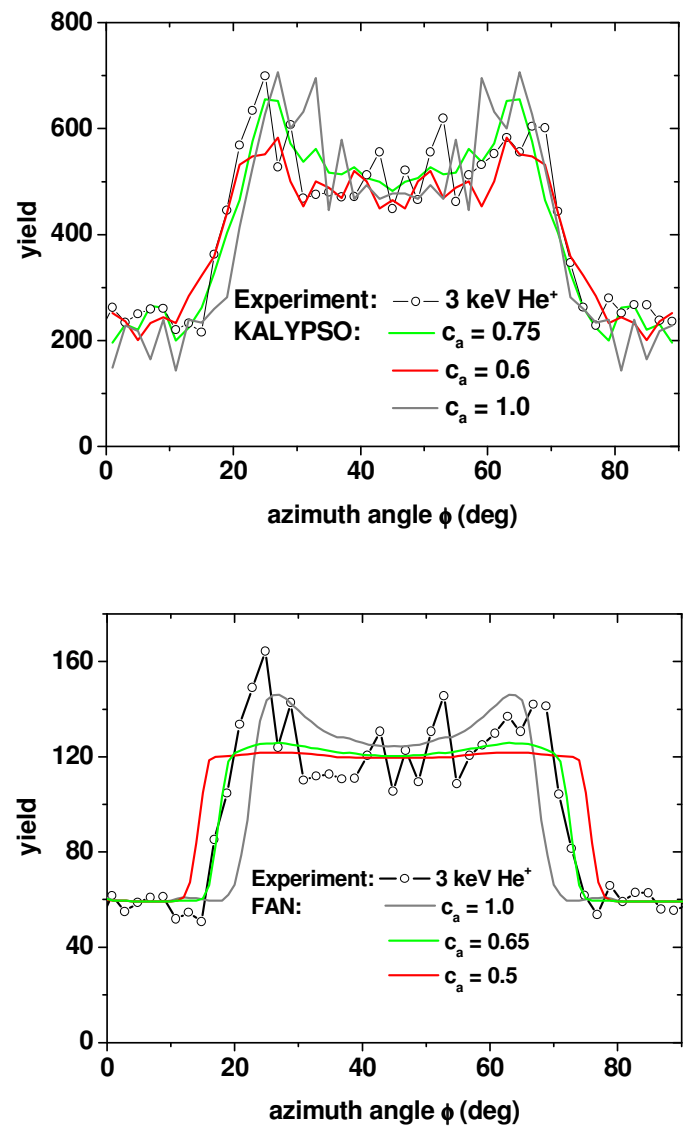

Fig.6: Azimuth scan obtained for $3 \mathrm{keV} \mathrm{He}^{+}$ scattered from $\mathrm{Cu}(100)$ and normal incidence (line + symbols). The experimental data was obtained by integration of an intermediate TOF-window with the width of the surface peak and subtraction of a linear decreasing background (see Fig1). KALYPSO simulations obtained for different interaction potential strength (lines).

Fig.7: Azimuth scan obtained for $3 \mathrm{keV} \mathrm{He}^{+}$ scattered from $\mathrm{Cu}(100)$ and normal incidence (line + symbols). The experimental data was obtained by integration of an intermediate TOF-window with the width of the surface peak and subtraction of a linear decreasing background (see Fig1). FAN simulations obtained for different interaction potential strength (lines).

By comparison of the presented experimental data to the different simulations it becomes obvious, that with background subtraction both features, the ratio of maximum to minimum height as well as the width of the maximum (FWHM) are obviously fit best by $\mathrm{c}_{\mathrm{a}}=0.75$ for MARLOWE and KALYPSO. These results are close to the values obtained in [8], [10], [19]. Furthermore, it has to be mentioned that MARLOWE and KALYPSO both well reproduce not only the width of the maximum but also the relative intensities of maximum and minima observed in the experimental dataset, i.e. they clearly reproduce the enhancement of intensity at the edge of the maximum caused by focusing of second layer scattered particles by first layer atoms. The results of both codes are quite similar for the investigated system and potential strengths. From that it can be claimed that KALYPSO offers an interesting alternative that may be preferable to MARLOWE for small targets due to its shorter computation time.

In FAN (Fig.7) the agreement is best for using a smaller correction factor $\mathrm{C}_{\mathrm{a}}=0.65$. As mentioned beforehand several differences of the results to the other codes are observed. The screening correction obtained from the angular position of the maximum is slightly reduced in comparison to the value deduced from using the more time consuming codes. However, FAN cannot reproduce the height ratios in a correct way and focusing effects are smaller. Fig. 8 presents simulations obtained by all three codes used for unity screening correction and the simple two-layer system. Again the coincidence of MARLOWE and KALYPSO is remarkable whereas FAN shows significant differences.

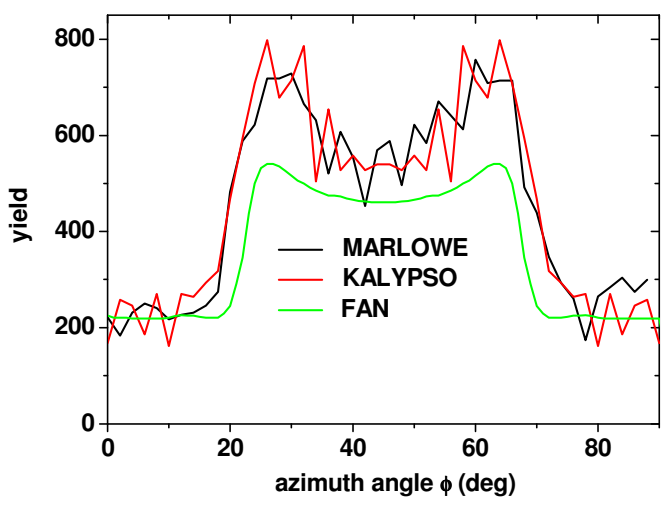

Fig.8: Azimuth scan of $3 \mathrm{keV} \mathrm{He}{ }^{+}$scattered from $\mathrm{Cu}(100)$ and normal incidence obtained from the different simulation codes for unity screening correction factor. Note the remarkable coincidence of MARLOWE and KALYPSO. FAN underestimates the height and width of the maximum (details see text).

The consistency of experimental and simulated spectra in Fig. 4 and 5 for $c_{a}=0.75$ and also Fig. 6 for $c_{a}=0.65$ further shows that single scattering trajectories from the first two monolayers can be selected. In fact, this means that for single crystalline targets a limitation of the information depth in charge integrated spectra to the outermost atomic layers is easily feasible. This is of relevance for quantitative surface composition analysis, e.g. accurate determination of ion fractions. It allows studying surface composition and structure of single crystals by evaluating charge integrated yields. Furthermore the absolute value of the interaction potential strength is of importance to interpret 
backscattered intensities properly. Fig. 9 finally shows the relative strength of the interatomic potential using different screening functions and screening length correction factors.

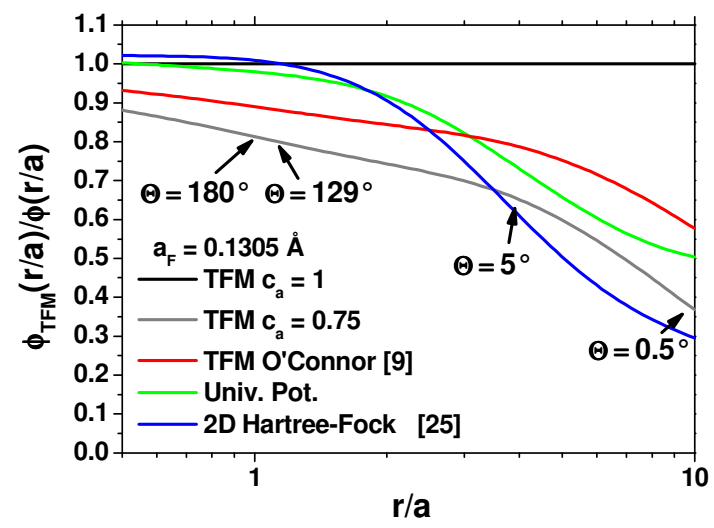

Fig.9: Comparison of different screening functions for Thomas-Fermi-Moliere and Universal potential in the range of interaction distances important in LEIS. The distances of closest approach associated with the identified scattering angles for $3 \mathrm{keV} \mathrm{He}^{+}$ ions are indicated.

It shows that our results correspond to a slightly weaker interaction than predicted by the Universal potential or the potential proposed by O'Connor [9]. For the specific interaction distance probed in our experiments the difference is found to be $20 \%$ and $10 \%$ respectively. The figure also shows the potential obtained by a 2D Hartree-Fock method [25].

A further important consequence of these findings is that one screening correction factor is applicable for description of the TFM scattering potential in the whole range of impact parameters needed to perform these simulations. Since simulations were carried out for a multilayer system and separately for the surface layers it can be assured that the obtained screening correction has no average character over a wide impact parameter range. Therefore, the scattering potential is suitable to describe single scattering from the surface as well as multiple small angle scattering. However, in order to perform reasonable simulations it is still necessary to know exactly what is the origin of the scattered contributions that are taken into account from the experimental spectra. If background contributions are included a rather large crystal (e.g. >>1000 atoms) has to be simulated which may lead to inconvenient computing times. Subtracting background contributions limits the system to the outermost atomic layers, which will enhance calculation speed and still deliver correct results.
To summarize, the choice of different simulation programs showed that they may deliver significantly different angular scans, even for identical crystal structures and scattering potentials. FAN simulations were found to result in systematically too low screening correction factors, and reproduce the overall shape of angular scans in an unsatisfying way. Nevertheless a quick estimate of the systems properties is possible by FAN if one knows about these pitfalls. KALYPSO was found to be an easy-to-handle alternative to the MARLOWE code since this code delivered concordant results for the model system investigated.

\section{Conclusions}

The aim of the present work was to elucidate why different approaches to extract information on the scattering potential from angular scans in LEIS may deliver inconsistent results. Experimental and simulated azimuth scans of $\mathrm{He}^{+}$scattered from $\mathrm{Cu}(100)$ were compared. Different evaluation procedures were investigated, with special emphasis on the background intensity due to contributions from deeper layers and due to multiple scattering. It was shown that limitation of the information to the topmost layers can also be achieved for charge integrated spectra. A distinct dependence of the obtained interaction potential strength on the evaluation method and the used simulation code was revealed. It was shown that one screening correction factor for the TFM potential is applicable for the whole range of impact parameters. This, however, needs coordinated spectrum evaluation and simulation input.

\section{Acknowledgment}

The authors acknowledge interesting and helpful communication with $M$. Karolewski. This work was partly supported by the Austrian Science Fund (FWF) under contract number P16469-N08. D. Primetzhofer acknowledges financial support due to a DOCfellowship by the Austrian Academy of Science.

[1] H.H. Brongersma, M. Draxler, M. de Ridder and P. Bauer, Surf. Sci. Rep. 62 (2007) 63.

[2] H. Niehus, W. Heiland, E. Taglauer, Surf.

Sci. Rep. 17. (1993) 213.

[3]N. P. Wang, Evelina A. García, R. Monreal, F. Flores, E. C. Goldberg, H. H. Brongersma, P. Bauer, Phys. Rev. A 64, (2001) 012901

[4] G. Molière, Z. Naturforsch. 2a (1947) 133.

[5] H. Winter, Phys. Rep. 367 (2002) 388.

[6] G.V. Dedkov, Phys. Stat. Sol. A 149 (1995) 453

[7] O.B. Firsov. Sov. Phys. JETP 6 (1958), 534. 
[8] E. Everhart, G. Stone and R.J. Carbone, Phys. Rev. 99 (1955) 1287.

[9] D.J. O'Connor and J.P. Biersack., Nucl. Inst. and Meth. B 15 (1986) 14

[10] Th. Fauster and M.H. Metzner. Surf. Sci. 166 (1986) 29.

[11] H. Niehus, R. Spitzl, Surf. Interface Anal. 17 (1991) 287.

[12] H. Niehus, FAN User Manual, Available from: http://asp2.physik.hu-berlin.de/main.html. [13] M.T. Robinson, I.M. Torrens, Phys. Rev. B 9 (1974) 5008.

[14] M.A. Karolewski, Nucl. Instr. Meth. B 230 (2005) 402

[15] M. Draxler, M. Walker, C.F. McConville, Nucl. Instr. Meth. B 249 (2006) 812

[16] Y. Yamamura, W. Takeuchi, T. Kawamura, Nucl. Instr. Meth. B 153 (1999) 71

[17] B. Poelsema, L.K. Verhey, A.L. Boers, Surf. Science 64, (1977) 554.

[18] S.T. Nakagawa, Radiat. Eff. 116 (1991) 21.

[19] R. Souda, M. Aono, C. Oshima, S. Otani, Y. Ishizawa, Surf. Sci. 176 (1986) 657.

[20] R. Beikler, E. Taglauer, Nucl. Instr. Meth. B 193 (2002) 455

[21] D. Primetzhofer, S.N. Markin, R. Kolarova, M. Draxler, R. Beikler, E. Taglauer, P.Bauer, Nucl. Instr. Meth. B 258 (2007) 36

[22] Landolt Börnstein, III/24/A Springer Verlag [23] M. Draxler, S.N. Markin, S.N. Ermolov, K. Schmid, C. Hesch, R. Gruber, A. Poschacher, M. Bergsmann, P. Bauer, Vacuum 73 (2004) 39.

[24] Stereographic projections of fcc-surfaces, Daresbury Laboratories http://www.dl.ac.uk/MEIS/stereographs/index.h $\mathrm{tm}$

[25] R. Andrzejewski, PhD thesis, Universidad Autónoma de Madrid (2008) 